\title{
Vidas conectadas: tecnología digital, interacción social e identidad
}

\author{
Javier SERRANO-PUCHE \\ Universidad de Navarra \\ jserrano@unav.es
}

\begin{abstract}
Resumen
Hoy en día las personas ya se relacionan tanto en el ámbito online como en el offline. Con la popularización de las tecnologías digitales, y especialmente de las redes sociales, el medio digital se ha constituido en un nuevo espacio de interacción. Basado en una amplia revisión bibliográfica, este artículo describe los rasgos de nuestra sociedad hiperconectada y analiza la influencia de la tecnología digital en la comunicación interpersonal y en la expresión de la identidad.
\end{abstract}

Palabras clave: Comunicación digital; Tecnología digital, Identidad; Facebook; Redes sociales; Interacción social

\section{Connected lives: digital technology, social interaction and identity}

\begin{abstract}
Nowadays people relate to each other both online and offline. With the popularization of digital technology, especially social networks, digital media has become a new realm of interaction. Based on a complete literature review, this paper describes the features of our hyper-connected society and it analyzes the impact of digital technology on interpersonal communication and self-expression.
\end{abstract}

Key words: Digital communication; Digital Technology; Identity; Social networks; Facebook; Social Interaction.

Referencia normalizada:

Serrano Puche, J. (2013) Vidas conectadas: tecnología digital, interacción social e identidad. Historia y Comunicación Social. Vol. 18 Nº Especial Noviembre. Págs. 353-364.

Sumario: 1. Introducción. 2. Vidas conectadas: tecnología y sociedad en red. 3. Interacción social y expresión identitaria en el ámbito digital. 4. Conclusiones. 5. Referencias bibliográficas

\section{Introducción}

Uno de los rasgos distintivos de nuestra época es la presencia constante y ubicua de las tecnologías digitales en la vida cotidiana, debido en gran medida a la proliferación de dispositivos móviles con acceso a Internet. Como señalan Rainie y Wellman (2012: 1-108), vivimos una "revolución triple": los individuos están ahora interco- 
nectados a través de redes -el "nuevo sistema operativo social"-; su plataforma de contacto es Internet; y pueden acceder a ella en todo momento y lugar, gracias a la comunicación móvil.

Aunque habitualmente a las tecnologías digitales se les denomine tecnologías de la información y la comunicación (TIC), es una visión reduccionista, pues -apunta Reig (2012) - la apropiación que de ellas hacen las personas las convierten también en tecnologías del aprendizaje y el conocimiento (TAC) y en tecnologías para el empoderamiento y la participación (TEP). Todo ello confluye en la configuración de un nuevo ecosistema comunicativo, en el que emergen nuevas prácticas comunicativas y de interacción social. Gracias a la tecnología digital la sociabilidad humana se extiende y amplia: las personas pueden ya relacionarse tanto en el ámbito offline como en el online.

La consolidación del medio online como un nuevo espacio, en el que la interacción y el elemento social cobran fuerza, origina que las relaciones mediadas por la tecnología puedan complementar y en ocasiones sustituir a la comunicación interpersonal cara a cara. A estas alturas debería ser superfluo aclarar que las interacciones que tienen lugar en el entorno digital -con frecuencia erróneamente calificadas como 'virtuales'- son tan reales como las que se llevan a cabo en el mundo presencial, aunque en ellas no haya la corporeidad que sí acompaña las relaciones en el mundo físico. De hecho, hoy en día asistimos a "una creciente normalización por la que la sociabilidad online forma parte cotidiana de la vida diaria de las personas; y (...) las relaciones sociales ya están hibridadas entre ambos contextos" (del Fresno, 2011: 93).

Una de las muestras más representativas de la estrecha vinculación que existe entre los entornos online y offline es el uso generalizado de las redes sociales digitales, que permiten tanto consolidar relaciones ya establecidas en el mundo presencial como construir nuevos lazos sociales. Son, por otra parte, un espacio privilegiado para la encarnación del "yo" en el ámbito digital, pues en ellas los usuarios publican características de sí mismos y comparten su actividad en Internet.

Al constituirse como un espacio de socialización y de expresión identitaria, una aproximación teórica hacia la esfera digital puede servirse inicialmente de las contribuciones más generales sobre la construcción de la identidad en nuestros días (Taylor, 1996; Bauman, 2007; Sibilia, 2008; Elliott, 2011). Incluso bibliografía clásica sobre comunicación interpersonal e identidad como La presentación de la persona en la vida cotidiana (Goffman, 1959) resulta sugerente para examinar cómo el "yo" se exhibe en el medio online. Como hemos señalado en otro lugar (Serrano-Puche, 2012), aunque el modelo dramatúrgico goffmaniano fuera concebido para situaciones de copresencia física, sus ideas son en gran medida también aplicables a las interacciones mediadas por la tecnología. Pese a todo, hay que subrayar que las peculiaridades del ámbito digital condicionan el modo en que el "yo" se despliega, y como tal es un fenómeno que reclama para su comprensión nuevas aproximaciones específicamente centradas en dicho entorno, tanto en el plano teórico-conceptual 
(Turkle, 1995; Fornas et al, 2002; Baym, 2010; Papacharissi, 2011) como metodológico (Kozinets, 2009; Hine, 2012, 2013).

En las páginas que siguen -y apoyándonos en una extensa revisión bibliográficaintentaremos: en primer lugar, proporcionar un breve marco teórico que ayude a comprender el impacto que tiene, en el seno de las sociedades contemporáneas, la tecnología digital en la comunicación interpersonal; y, a continuación, presentar los rasgos características de la interacción social y la expresión de la identidad personal en el ámbito online.

\section{Vidas conectadas: tecnología y sociedad en red}

Gilles Lipovetsky y Jean Serroy ofrecen un diagnóstico acertado de nuestro tiempo cuando afirman que "vivir es, de manera creciente, estar pegado a la pantalla y conectado a la red" (2009: 271). Por un lado, podemos en efecto constatar que la nuestra es una "sociedad multipantalla" (Pinto, 2008), que hace de ellas un intermediario casi inevitable en las relaciones con el mundo y con los demás. Paralelamente, la hiperconectividad se ha consolidado como un rasgo distintivo de esta época, donde prima la cultura de la velocidad y la eficiencia, y en la que hay que lidiar con la sobreabundancia de información, que transita prioritariamente a través de círculos sociales.

Por otra parte, las peculiaridades del medio digital (caracterizado por su arquitectura en red, donde unas interacciones/nodos llevan a otras) facilitan una flexibilidad cada vez mayor en las relaciones entre individuos y grupos. Asistimos al desarrollo de un "individualismo reticular" (Wellmann et al, 2003), pues "aunque no renunciamos a las relaciones de pertenencia, tendemos a multiplicar las relaciones reticulares transitorias de alcance limitado, menos rígidas y más dinámicas" (Pisani y Piotet, 2009: 63). En las interacciones mediadas por la tecnología, la persona puede controlar en mayor medida que en los encuentros cara a cara el grado de implicación que quiere asumir, puesto que no se ve interpelada ni por las exigencias que comporta la corporeidad ni por la simultaneidad temporal que sí existen en las interacciones del mundo físico. En este sentido, y aunque en buena medida dependerá de la actitud de cada persona, es cierto que las interacciones digitales no tienden de por sí a facilitar "una comunicación a la vieja usanza, en la que se comprometía mucho el yo, sino una comunicación efímera y fragmentaria, cambiante y removible a la manera en que la cultura de consumo ha enseñado a adquirir" (Verdú, 2012). Este modo 'saneado' de relacionarse, estando en compañía sin renunciar al mismo tiempo a la individualidad (Turkle, 2011), se ajusta bien al mundo "líquido" en el que vivimos (Bauman, 2007). Como señala Gómez Cabranes (2013: 231) "Internet, como 'tecnología de identidad' donde es posible experimentar diversos yoes, confluye con la identidad posmoderna: nos induce a pensarnos como "fluidos, emergentes, descentralizados, múltiples y constantemente en proceso' (Turkle, 1995: 263-264)". 
Internet es una tecnología de identidad y es, sobre todo, una tecnología de conexión. Por eso, y especialmente entre los jóvenes, la conectividad permanente se ha convertido hoy en día en un elemento esencial a la hora de construir y manejar las amistades y la vida social (Moeller, Powers y Roberts, 2012; Haaf, 2012: 41-75). No obstante, ese uso demasiado intenso de las tecnologías digitales puede provocar en las personas una tensión entre el deseo de estar unidos a través de la tecnología y al mismo tiempo sentirse atrapados por ella (Baron, 2011; Hall y Baym, 2012). En este sentido, algunas investigaciones señalan que los jóvenes muestran un gran interés por saber de los demás, hasta el punto de sufrir el "síndrome FOMO" (Fear Of Missing Out): no pueden privarse de usar Internet, por el miedo a estar perdiéndose algo (Vaughn, 2012). Paralelamente, con frecuencia piensan que si dejan de enviar mensajes corren el riesgo de volverse invisibles frente a sus contactos. Como apunta Rosalía Winocur (2012: 80) "estar conectado implica esencialmente estar visible. La visibilidad garantiza la inclusión en un mundo cuya representación se ha desplazado de lo palpable a lo comunicable. La clave que explica lo trascendente que se ha vuelto estar visible radica en lo amenazadora que resulta la invisibilidad. En términos de trascendencia social, para los jóvenes lo que no puede ser visto en los medios o subido a la Red no existe". De alguna manera parece advertirse, pues, que el deseo de estar continuamente conectado -y de encontrar en la mirada de los otros la confirmación de la propia existencia- gana peso frente a la introspección y a la voluntad de crecer para adentro. "La soledad, la búsqueda y el desarrollo de un espacio propio, de una interioridad rica y frondosa, es un proyecto en proceso de abandono -señala Caro Castaño (2012: 63) - De ahí que la falta de conexión con los otros devenga en problema: no hay una interioridad valiosa en la que refugiarse".

Todo ello conduce a una redefinición de las fronteras entre lo que es considerado privado y lo que es público (West, Lewis y Currie, 2009; Brandtzaeg, Lüders y Skjetne, 2010). La intimidad no desaparece como tal, ni deja de ser una cuestión que preocupe a los usuarios de las tecnologías digitales (Boyd y Hargittai, 2010; Madden, 2012), pero sí sufre un reajuste en su sentido y alcance, porque "cada vez es más difícil encapsular una información que es fluida por naturaleza y fluye, aún más, en los ecosistemas de redes digitales (...) Si antes decidíamos qué aspectos de nuestra vida convertíamos en públicos, ahora (...) debemos decidir qué preservar y trabajar de forma activa para lograrlo" (Reig, 2013: 64).

\section{Interacción social y expresión identitaria en el ámbito digital}

Como ya ha sido señalado, la tecnología digital ha traído consigo una ampliación de los espacios para el desarrollo de la sociabilidad humana, permitiendo así nuevas vías de comunicación e interacción social. Éstas pueden asumir una gran diversidad de formas pues, como indica Gómez Cabranes, "Internet no es un medio tecnológico más, sino que constituye además una mediación sociocultural, con distintos entornos sociotécnicos" (2013: 219). Factores como las posibilidades expresivas de cada de 
uno de esos entornos, los temas y tópicos que centran la interacción, el contexto y propósito de uso de las personas, su grado de anonimato o autorrevelación y la inversión de tiempo o frecuencia con que se conectan determinan el tipo y alcance de dichas interacciones.

Dentro del ámbito digital, es en las redes sociales donde la capacidad de expresión comunicativa por parte de los usuarios puede llegar a ser más rica y compleja (aunque en diferentes grados, dadas las notables diferencias que existen entre unas y otras). La popularización de su uso da forma a un fenómeno en auge: la Mass self-communication, en palabras de Castells (2009).

En toda red social el usuario siempre dispone de un "perfil", a modo de corporeización digital (Boyd y Heer, 2006), para que el resto de usuarios pueda identificarle e interactuar con él. En dicho perfil, "la foto vale por la persona" (Illouz, 2007: 174), ya que ésta la escoge en función de cómo quiere ser percibido por los demás usuarios: para alcanzar la aceptación social, intentará aparecer del mejor modo posible, ya sea realzando la faceta de la sociabilidad, el buen humor, el atractivo físico o las capacidades deportivas. Son ésas las más recurrentes a la hora de autopresentarse en las fotos de perfil en las redes sociales, tal como señalan los análisis de contenido realizados por Siibak (2009), Strano (2008), Hum y colaboradores (2011) o Farquhar (2013). Al mismo tiempo, en la proyección de sí mismo que ofrece el individuo con frecuencia suele existir una tensión latente entre su verdadera imagen y una imagen idealizada del yo (Manago et al, 2008; Back et al, 2010). Esa tensión está más acentuada en el caso de los sitios webs de citas-donde la finalidad última es trasladar la interacción al plano presencial-, pues el deseo de ser atractivo se ve constreñido por las exigencias de autenticidad y el miedo a decepcionar al otro en un posible encuentro cara a cara. No obstante, es comúnmente aceptado entre los usuarios de estas redes cierto margen de discordancia entre la realidad y la imagen fotográfica que presenta el usuario ya que, más que una representación exacta del yo offline, el perfil del usuario "constituye una promesa hecha a un público imaginario de que una futura interacción cara a cara se llevaría a cabo con una persona que no difiere fundamentalmente de la persona representada por el perfil" (Ellison, Hancock y Toma, 2012: 56).

Así pues, cabe constatar que en el espacio digital -en mayor medida que cuanto sucede en las situaciones de copresencia física- un componente esencial de la comunicación es la 'gestión de las impresiones' (Goffman, 1959) que la persona quiere causar en sus interlocutores. Ese manejo de las impresiones a la hora de construir y expresar la identidad personal se desarrolla tanto de un modo narrativo-verbal como, sobre todo, de manera visual (Zhao, Grasmuck y Martin, 2008); y dado el tipo de sociabilidad característico del medio digital la self-expression a menudo adquiere rasgos de una self-promotion (van Dijck, 2013). A este respecto, son numerosas las investigaciones que demuestran la relevancia de los elementos del perfil como factor determinante en el tipo y calidad de interacciones que tendrá el usuario en esa red social. Lampe, Ellison y Steinfield (2007) detectaron en un estudio de más de 30.000 perfiles de Facebook que existía una relación directa entre la cantidad y el nivel de concreción de la información disponible en los perfiles de los usuarios y el número 
de contactos conectados a ellos. Yendo más allá, Walther y colaboradores (2008) y Utz (2010) han demostrado que el número y el grado de atractivo de los contactos que tiene el usuario influyen en la manera en que dicho usuario es percibido por los demás. En la misma línea, otras investigaciones (Tong et al, 2008; Zywica y Danowski, 2008) apuntan a que una sobreabundancia de "amigos" puede transmitir una imagen negativa del usuario; que éste sea visto como alguien demasiado dependiente de las interacciones digitales o deseoso por alcanzar una popularidad de la que probablemente carezca en el mundo presencial.

A diferencia de la comunicación offline, donde hay presencia física, el ámbito digital permite que uno construya una imagen de sí mismo con mucha mayor facilidad y rapidez. En circunstancias normales de interacción -entendiendo por éstas aquellas en las que la persona no pretende asumir una identidad falsa ni enmascarar de modo radical su personalidad-, habrá elementos que siempre le acompañen (los vinculados directamente con su identidad real básica, como el sexo, edad o raza), mientras que otros podrán sufrir variaciones en función del escenario, es decir, de la red social concreta en la que el actor representa su personaje, por decirlo en términos goffmanianos. El usuario puede proyectarse simultáneamente en diferentes espacios de interacción sin que las imágenes de su "yo" sean siempre coherentes entre sí, incluso pueden diferir sustancialmente de la auténtica identidad personal (Arcila, 2011). Por tanto, es más fácil llevar a cabo operaciones de modificación y ocultamiento de la identidad, acciones que van adquiriendo cierto estatus de normalidad entre los jóvenes, sobre todo en interacciones ligadas al entretenimiento y la diversión (Cáceres, Ruiz San Román y Brändler, 2009). En esos casos se constata las limitaciones de la comunicación digital y los riesgos que conlleva la ausencia de corporeidad en las interacciones (con la consiguiente falta de pistas visuales y no verbales que sí están presentes en los encuentros no mediados por la tecnología). No obstante, dado que la interacción comunicativa siempre es el resultado del encuentro entre la expresión y la interpretación (Martín Algarra, 2003: 135-156), también en el entorno online se puede desentrañar de manera crítica la 'puesta en escena' del otro y rebajar sus pretensiones autorrepresentativas, ya sea porque uno conoce a aquella persona fuera de las redes sociales y es capaz de comparar el 'personaje representado' con su correspondiente identidad real, ya sea porque percibe facetas identitarias disonantes e involuntarias en su modo de presentarse ante los demás.

Junto con la información dada inicialmente al crear su perfil en la red social, la persona continúa expresando su identidad por medio de su actividad online. Cada vez que actualiza su "estado" (ya sea con comentarios originales, frases célebres, videoclips, anuncios, recomendación de enlaces -noticias y contenidos de marca-, inclusión de nuevas fotos personales, etc.) está ofreciendo una 'performance' de sus gustos y aficiones (Liu, 2007). Por decirlo con la metáfora de Caro Castaño (2012), la "identidad mosaico" del usuario se va componiendo con "teselas" de autoría, temática y forma diversa, al combinar en sus interacciones digitales contenidos originales y propios con otros contenidos de la industria y la cultura popular. 
Entre las investigaciones sobre identidad y redes sociales otra cuestión recurrente es aquella que alude a los efectos y consecuencias psicológicas que tiene el empleo frecuente de las tecnologías digitales. Algunos tópicos analizados son la relación entre la intensidad en el uso de las redes y la autoestima personal (Valkenbur, Peter y Schouten, 2006; Gonzales y Hancock, 2011; Tazghini y Siedlecki, 2013), las disonancias entre la presentación online del "yo" y la presentación offline (DeAndrea y Walther, 2011) o la tendencia al narcisismo entre los usuarios jóvenes (Buffardi y Campbell, 2008; Twenge y Campbell, 2009; Ong et al, 2011). A menudo, las conclusiones de esos estudios suelen apuntar a un impacto netamente positivo o negativo de la Red en las personas, como si aquella fuera un constructo unidimensional. En este punto, estamos de acuerdo con Omar Rosas (2012: 216) en que "dada la variedad y complejidad de las actividades relacionadas con Internet, parece claro que los diferentes modos de usar Internet tienen potencial para producir tanto daños como beneficios psicológicos en los usuarios".

También se estudia desde el punto de vista sociológico si una sobrexposición desmedida en el ámbito digital puede propiciar que los usuarios desdeñen la socialización directa en el espacio físico, provocando su aislamiento respecto a su entorno inmediato (Sum et al, 2008). En efecto, "la comunicación online tiene más intensidad e inmediatez y es, en cierto modo, más adictiva que la presencial (...) Este tipo de comunicación, unido a la velocidad e intensidad del contacto da lugar a una cierta 'hipercomunicación', lo que parece venir facilitado por la naturaleza informal y desinhibidora del medio" (Gómez Cabranes, 2013: 227). Sin embargo, y como han señalado otras investigaciones (Boase et al, 2006; Rainie, Purcell y Smith, 2011; Hampton et al, 2011), las interacciones digitales ayudan más bien a mantener y consolidar la variedad de vínculos sociales previamente creados en el mundo presencial (fuertes, débiles, de carácter instrumental, emocional, afiliativo, etc.). El uso frecuente de Internet parece repercutir positivamente en la implicación del usuario en su ámbito social, y no al contrario.

\section{Conclusiones}

La consolidación de las tecnologías digitales en las diferentes situaciones y actividades diarias ha propiciado que ya no vivamos "con" los medios de comunicación, sino más bien "en" los medios (Deuze, 2012). Internet ha adquirido una relevancia indudable como nuevo ámbito para la comunicación interpersonal, marcando un antes y un después en las prácticas comunicativas y en la interacción social (Jordan, 2013). Las personas tienen ahora en el entorno digital un nuevo espacio para su desarrollo personal y para la socialización, que se suma al contexto social tradicional, aunque presenta particularidades propias, dada su condición tecnológica.

En este sentido, las características del ámbito digital -en el que las interacciones no necesitan de un encuentro cara a cara ni de la sincronización temporal- potencian la 
capacidad de la persona de presentar su identidad de manera controlada y selectiva, pudiendo decidir qué, cómo, cuánto y cuándo revela de su "yo". Este networked self (Papacharissi, 2011), aun teniendo una esencia nuclear, enfatiza determinados aspectos de su identidad (ocultando quizá otros), en función del entorno concreto en el que interactúa, ya que muchos de estos contactos interpersonales están basados en atributos particulares del individuo, no en toda la personalidad como tal. Por otra parte, cabe constatar la creciente normalización y enriquecimiento mutuo de las esferas presencial y digital en la socialización cotidiana de las personas.

\section{Referencias bibliográficas}

ARCILA, C. (2011). "Análisis comparativo de la presentación personal virtual en diferentes espacios de interacción". En: Fonseca Journal of Communication, $\mathrm{n}^{\circ}$ 3, pp. 153-169.

BACK, M. et al (2010). "Facebook profiles reflect actual personality, not self-idealization". En: Psychological Science, XX, pp. 1-3.

BARON, N. S. (2011). "Concerns about mobile phones: A cross-national study". En First Monday, vol 16, $\mathrm{n}^{\circ} 8-1$ August. Disponible en: http://firstmonday.org/htbin/ cgiwrap/bin/ojs/index.php/fm/article/view/3335/3032 [29-07-2013].

BAUMAN, Z. (2007). Tiempos líquidos: vivir en una época de incertidumbre. Barcelona: Tusquets.

BAYM, N. K. (2010). Personal connections in the digital age. Cambridge: Polity Press.

BOASE, J. et al (2006). The Strenght of Internet Ties. Pew Research Center's Internet \& American Life Project. Disponible en: http://www.pewinternet.org/ Reports/2006/The-Strength-of-Internet-Ties.aspx [08-11-2012].

BOYD, D.; HARGITTAI, E. (2010). "Facebook privacy settings: Who cares?". En: First Monday, vol 15, $\mathrm{n}^{\mathrm{o}}$ 8-2 August. Disponible en: http://firstmonday.org/ojs/ index.php/fm/article/view/3086 [07-10-2013].

BOYD, D.; HEER, J. (2006). "Profiles as conversation: Networked identity performance on Friendster". En: Proceedings of the Thirty-Ninth Hawai'i International Conference on System Sciences. Los Alamitos, CA: IEEE Press.

BRANDTZAEG, P; LÜDERS, M.; SKJETNE, J. H. (2010). "Too Many Facebook "Friends"? Content Sharing and Sociability Versus the Need for Privacy in Social Network Sites". En: International Journal of Human-Computer Interaction, 26 (11-12), pp. 1006-1030.

BUFFARDI, L. E.; CAMPBELL, W. K. (2008). "Narcissism and Social Networking Web Sites". En: Personality and Social Psychology Bulletin, vol. 34, no 10, october, pp. 1303-1314.

CÁCERES, M. D.; RUIZ SAN ROMÁN, J. A.; BRÄNDLE, G. (2009). "Comunicación interpersonal y vida cotidiana. La presentación de la identidad de los jóvenes en Internet". En: CIC, Cuadernos de Información y Comunicación, vol. 14, pp. 213-231. 
CARO CASTAÑO, L. (2012). "Identidad mosaico. La encarnación del yo en las redes sociales digitales". En: TELOS: cuadernos de comunicación e innovación, $\mathrm{n}^{\mathrm{o}}$ 91, abril-junio, pp. 59-68.

CASTELLS, M. (2009). Communication power. Oxford: Oxford University Press.

DEANDREA, D.; WALTHER, J. B. (2011). "Attributions for Inconsistencies Between Online and Offline Self-Presentations". En: Communication Research, 38 (6), pp. 805-825.

DEL FRESNO, M. (2011). Netnografia. Barcelona: Editorial UOC.

DEUZE, M. (2012). Media Life. Cambridge: Polity Press.

ELLIOTT, A. (2011) (ed). Routledge Handbook of Identity Studies. New York: Routledge.

ELLISON, N.; HANCOCK, J.; TOMA, C. L. (2012). "Profile as promise: A framework for conceptualizing veracity in online dating self-presentations". En: New Media \& Society, 14 (1), pp. 45-62.

FARQUHAR, L. (2013). "Performing and interpreting identity through Facebook imagery". En: Convergence, 19 (4), pp. 446-471.

FORNAS, J. et al (eds) (2002). Digital Borderlands: Cultural Studies of Identity and Interactivity on the Internet. New York: Peter Lang.

GOFFMAN, E. (1959). The Presentation of Self in Everyday Life. New York: Doubleday Anchor Books.

GÓMEZ CABRANES, L. (2013). "Las emociones del internauta". En: FLAMARIQUE, L.; D'OLIVEIRA-MARTINS, M. (2013) (eds.). Emociones y estilos de vida: radiografía de nuestro tiempo. Madrid: Biblioteca Nueva, pp. 211-243.

GONZALES, A. L.; HANCOCK, J. T. (2011). "Mirror, Mirror on my Facebook Wall: Effects of Exposure to Facebook on Self-esteem". En: Cyberpsychology Behavior Social Networking, 14 (1-2), pp. 79-83.

HAAF, M. (2012). Dejad de lloriquear. Sobre una generación y sus problemas superfluos. Barcelona: Alpha Decay.

HALL, J. A.; BAYM, N. K. (2012). "Calling and texting (too much): Mobile maintenance expectations, (over) dependence, entrapment, and friendship satisfaction". En: New Media \& Society, 14 (2), pp. 316-331.

HAMPTON, K. N., et al (2011). Social Networking Sites and Our Lives: How People's Trust, Personal Relationships, and Civic and Political Involvement are Connected to Their Use of Social Networking Sites and Other Technologies. Washington, D.C.: Pew Research.

HINE, C. (2013). Virtual Research Methods (Four Volume Set). London: Sage. (2012). The Internet. Understanding Qualitative Research. London: Oxford.

HUM, N. et al (2011). "A picture is worth a thousand words: A content analysis of Facebook profile photographs". En: Computers in Human Behavior, 27 (5), pp. 1828-1833.

ILLOUZ, E. (2007). Intimidades congeladas. Las emociones en el capitalismo. Buenos Aires: Kazt Editores.

JORDAN, T. (2013). Internet, Society and Culture. Communicative Practices Before and After the Internet. New York-London: Bloomsbury. 
KOZINETS, R. V. (2010). Netnography. Doing Ethnographic Research Online. London: Sage.

LAMPE, C.; ELLISON, N.; STEINFIELD, C. (2007). "A familiar Facebook: Profile elements as signals in an online social network". En: VV.AA. Proceedings of Conference on Human Factors in Computing Systems. New York: ACM Press, pp. 435-444.

LIPOVETSKY, G.; SERROY, J. (2009). La pantalla global. Cultura mediática y cine en la era hipermoderna. Barcelona: Anagrama.

LIU, H. (2007). "Social network profiles as taste performances". En: Journal of Computer-Mediated Communication, 13 (1), pp. 252-275.

MADDEN, M. (2012). Privacy managment on social media sites. Pew Research Center's Internet \& American Life Project. Disponible en: http://www.pewinternet.org/Reports/2012/Privacy-management-on-social-media.aspx [07-10-2013].

MANAGO, A. M. et al (2008). "Self-presentation and gender on MySpace". En: Journal of Applied Developmental Psychology, 29, pp. 446-458.

MARTÍN ALGARRA, M. (2003). Teoría de la comunicación: una propuesta. Madrid: Tecnos.

MOELLER, S.; POWERS, E.; ROBERTS, J. (2012). “"El mundo desconectado” y '24 horas sin medios': alfabetización mediática para la conciencia crítica de los jóvenes". En: Comunicar, 39, pp. 45-52.

ONG, E. et al (2011): "Narcissism, Extraversion and Adolescents' Self-presentation on Facebook". En: Personality and Individual Differences, 50 (2), pp. 180-185.

PAPACHARISSI, Z. (ed.) (2011). A Networked Self: Identity, Community, and Culture on Social Network Sites. New York: Routledge.

PINTO, M. (2008). "Investigating Information in the Multiscreen Society: An Ecologic Perspective". En: RIVOLTELLA, P. C. (ed.): Digital Literacy: Tools and Methodologies for Information Society. Hershey: IGI Global, pp. 207-216.

PISANI, F.; PIOTET, D. (2009). La alquimia de las multitudes: cómo la web está cambiando el mundo. Barcelona: Paidós.

RAINIE, L.; WELLMAN, B. (2012). Networked. The New Social Operating System. Cambridge, MA: The MIT Press.

RAINIE, L.; PURCELL, K.; SMITH, A. (2011). The Social Side of the Internet. Pew Research Center's Internet \& American Life Project. Disponible en: http://www. pewinternet.org/Reports/2011/The-Social-Side-of-the-Internet.aspx [09-062013].

REIG, D. (2013). "Describiendo al hiperindividuo, el nuevo individuo conectado". En REIG, D.; VÍLCHEZ, L. (2013). Los jóvenes en la era de la hiperconectividad: tendencias, claves y miradas. Madrid: Fundación Telefónica, pp. 23-90. (2012). "Disonancia cognitiva y apropiación de las TIC". En: TELOS: cuadernos de comunicación e innovación, $\mathrm{n}^{\circ}$ 90, enero-marzo, pp. 9-10.

ROSAS, O. (2012). "Types of Internet Use, Well-Being, and the Good Life. Ethical Views for Prudencial Psychology". En: BREY, P.; BRIGGLE, A.; SPENCE, E. (eds) (2012). The Good Life in a Technological Age. London: Routledge. 
SERRANO-PUCHE, J. (2012). "La presentación de la persona en las redes sociales: una aproximación desde la obra de Erving Goffman". En: Revista Anàlisi, n 46, septiembre, pp. 1-17.

SIBILIA, P. (2008). La intimidad como espectáculo. Buenos Aires: Fondo de Cultura Económica.

SIIBAK, A. (2009). "Constructing the Self through the Photo selection. Visual Impression Management on Social Networking Websites". En: Cyberpsychology: Journal of Psychosocial Research on Cyberspace, 3(1), article 1. Disponible en: http://cyberpsychology.eu/view.php?cisloclanku=2009061501\&article=1 [09-102013].

STRANO, M. M. (2008). "User descriptions and interpretations of self-presentation through Facebook profile images". En: Cyberpsychology: Journal of Psychosocial Research on Cyberspace, 2 (2), article 5. Disponible en: http://cyberpsychology.eu/view.php?cisloclanku=2008110402\&article $=5$ [09-10-2013].

SUM, S. et al (2008). "Internet Use and Loneliness in Older Adults". En: CyberPsychology and Behavior, 11 (2), pp. 208-211.

TAYLOR, C. (1996). Fuentes del yo: la construcción de la identidad moderna. Barcelona: Paidós.

TAZGHINI, S.; SIEDLECKI, K. (2013). "A mixed method approach to examining Facebook use and its relationship to self-esteem". En: Computers in Human Behavior, 29 (3), pp. 827-832.

TONG, S. T. et al (2008). "Too much of a good thing? The relationship between number of friends and interpersonal impressions on Facebook". En: Journal of Computer-Mediated Communication, 13 (3), pp. 531-549.

TURKLE, S. (1995). Life on the Screen: Identity in the Age of the Internet. New York: Simon \& Schuster.

(2011). Alone together. Why We Expect More from Technology and Less from Each Other. New York: Basic Books.

TWENGE, J. M.; CAMPBELL, W. K. (2009). The Narcissism Epidemic: Living in the Age of Entitlement. New York: Free Press.

UTZ, S. (2010): "Show me your friends and I will tell you what type of person you are: How one's profile, number of friends, and type of friends influence impression formation on social network sites". En: Journal of Computer-Mediated Communication, 15 (2), pp. 314-335.

VALKENBURG, P. M.; PETER, J.; SCHOUTEN, A. P. (2006). "Friend networking sites and their relationship to adolescents' well-being and social self-esteem. En: Cyberpsychology \& Behavior, 9 (5) p. 584-590.

VAN DIJCK, J. (2013). "'You have one identity': performing the self on Facebook and LinkedIn". En: Media, Culture \& Society, 35 (2), pp. 199-215.

VERDÚ, V. (2012). "El personismo". El Pais, 28-04-2012.

WALTHER, J. B. et al (2008). 'The role of friends' appearance and behavior on evaluations of individuals on Facebook: Are we known by the company we keep?”. En: Human Communication Research, 34 (1), pp. 28-49. 
WELLMAN, B. et al (2003). "The social affordances of the Internet for networked individualism". En: Journal of Computer-Mediated Communication, 8 (3).

WEST, A.; LEWIS, J.; CURRIE, P. (2009). "Students' Facebook 'friends': public and private spheres". En: Journal of Youth Studies, 12 (6), pp. 615-627.

WINOCUR, R. (2012). "La intimidad de los jóvenes en las redes sociales. Transformaciones en el espacio público y privado". En: TELOS: cuadernos de comunicación e innovación, $\mathrm{n}^{\circ}$ 91, abril-junio, pp. 79-88.

ZHAO, S., GRASMUCK, S.; MARTIN, J. (2008). "Identity construction on Facebook: Digital empowerment in anchored relationships". En: Computers in Human Behavior, 24 (5), pp. 1816-1836.

ZYWICA, J.; DANOWSKI, J. (2008). "The faces of Facebookers: Investigating social enhancement and social compensation hypotheses; predicting Facebook and offline popularity from sociability and self-esteem, and mapping the meanings of popularity with semantic networks". En: Journal of Computer-Mediated Communication, 14 (1), pp. 1-34.

\section{El autor}

Javier Serrano-Puche es Profesor Contratado Doctor en la Universidad de Navarra e investigador del Center for Internet Studies and Digital Life de dicha universidad. $\mathrm{Su}$ investigación actual se centra en comprender cómo la tecnología digital afecta al modo de comunicarnos, originando nuevas formas de interacción social. Especial atención le merecen las redes sociales y la comunicación a través de los móviles. Sus publicaciones más recientes han aparecido en revistas como "Communication \& Society / Comunicación y Sociedad", "Estudios sobre el Mensaje Periodístico", "El profesional de la información" o "Análisi". Este trabajo también se enmarca en el proyecto de investigación "Cultura Emocional e Identidad", integrado en el Instituto Cultura y Sociedad (ICS) de la Universidad de Navarra, del que el autor forma parte. 